\title{
Ocular iontophoresis of EGP-437 (dexamethasone phosphate) in dry eye patients: results of a randomized clinical trial
}

This article was published in the following Dove Press journal:

Clinical Ophthalmology

I3 May 201 I

Number of times this article has been viewed

\author{
Michael A Patane' \\ Amy Cohen' \\ Stephen From' \\ Gail Torkildsen ${ }^{2}$ \\ Donna Welch ${ }^{3}$ \\ George W Ousler III \\ 'Eyegate Pharmaceuticals, Inc, \\ Waltham, MA, USA; ${ }^{2}$ Andover Eye \\ Associates, Andover, MA, USA; \\ ${ }^{3}$ Ora, Inc, Andover, MA, USA
}

Correspondence: Michael A Patane Eyegate Pharmaceuticals, Inc, I00 Beaver Street, Waltham, MA 02453, USA

Tel + I 78|-788-9045

Fax +I 78I-788-9047

Email mpatane@eyegatepharma.com
Purpose: To assess safety and efficacy of EGP-437 (dexamethasone phosphate $40 \mathrm{mg} / \mathrm{mL}$ [DP]) in dry eye patients.

Methods: The study employed a prospective, single-center, double-masked design utilizing a Controlled Adverse Environment (CAE). Patients $(n=103)$ with confirmed signs and symptoms of dry eye syndrome were randomized into 1 of 3 iontophoresis treatment groups: $7.5 \mathrm{~mA}$ min at $2.5 \mathrm{~mA}$ (DP 7.5, $\mathrm{n}=41$ ); $10.5 \mathrm{~mA}$-min at $3.5 \mathrm{~mA}$ (DP 10.5, $\mathrm{n}=37$ ); or $10.5 \mathrm{~mA}$-min at $3.5 \mathrm{~mA}$ (placebo, $\mathrm{n}=25$ ). Three CAE visits and 4 follow-up visits occurred over 3 weeks. Patients meeting enrollment criteria received iontophoresis in both eyes after the second CAE exposure (visit 3) and before the third CAE exposure (visit 5). Primary efficacy endpoints were corneal staining and ocular discomfort. Secondary endpoints included tear film break-up time, ocular protection index (OPI), and symptomatology.

Results: The DP 7.5 and DP 10.5 treatment groups showed statistically significant improvements in signs and symptoms of dry eye at various time points; however, the primary endpoints were not achieved. The DP 7.5 treatment group exhibited statistically significant improvements in corneal staining (when comparing the differences between study entry and exit, 3 weeks, $P=0.039$ ), OPI (immediately following the second treatment, $P=0.048$ ) and ocular discomfort at follow-up visits (a week after the first treatment, $P=0.032 ; 24$ hours after the second treatment, $P=0.0032$ ). Treatment-emergent adverse events (AEs) were experienced by $87 \%$ of patients and were consistent across all treatment groups. Most AEs were mild and no severe AEs were observed.

Conclusion: Ocular iontophoresis of EGP-437 demonstrated statistically and clinically significant improvements in signs and symptoms of dry eye syndrome within a CAE model.

Keywords: iontophoresis, dry eye, Controlled Adverse Environment (CAE), ocular protection index (OPI)

\section{Introduction}

Dry eye syndrome is a "disorder of tear film deficiency, which leads to damage to the interpalpebral ocular surface and symptoms of ocular discomfort". ${ }^{1}$ Prevalence estimates of the disease range from $5 \%$ to $35 \%$ of individuals worldwide over age 50 years, with an estimated 5 to 6 million Americans reporting experiences of significant dry eye symptomatology. ${ }^{2}$

Patients with milder forms of dry eye often achieve transient, symptomatic relief from using over-the-counter topical ophthalmic formulations. These products can replenish the tear film and normalize the elevated tear osmolarity often associated with dry eye, but they do not treat the underlying cause of the disease. 
Chronic forms of dry eye require more intensive, longer-lasting therapeutic interventions. US Food and Drug Administration (FDA)-approved treatments include biodegradable inserts that act as prolonged-release ocular surface lubricants, and punctal plugs, which can slow tear film drainage. However, punctal plug users may experience spontaneous extrusion or migration of plugs, as well as a counterproductive reduction in tear secretion due to reduced drainage. ${ }^{3}$

Restasis $^{\circledR}(0.05 \%$ topical suspension, Allergan Inc, Irvine, CA) is the only FDA-approved drug for dry eye; specifically, it is indicated for patients with ocular inflammation due to keratoconjunctivitis sicca. ${ }^{4}$ While the exact mechanism of action is unknown, it is thought to act by stimulating tear production. ${ }^{4}$ Restasis has proven to be an effective treatment for only $15 \%$ of patients treated; ${ }^{4,5}$ therefore, the need for effective therapies to treat dry eye disease remains substantial.

The lack of available drug treatments for dry eye disease may be related to the multifaceted nature of the disease, both in terms of the causes and manifestations. These factors complicate therapeutic target identification. ${ }^{6}$ Growing scientific evidence obtained from studying a broad range of dry eye patients suggests that inflammation is either an underlying component or a primary cause of the tear film disruption; therefore, anti-inflammatory therapy is likely to be efficacious in a diverse patient population..$^{7-9}$

Topical corticosteroids are used off-label to reduce signs and symptoms of dry eye. ${ }^{10}$ These drugs suppress inflammation through multiple pathways. ${ }^{11}$ While corticosteroid eyedrops are widely used to treat dry eye, ${ }^{12}$ their low ocular bioavailability (estimated to be $1 \%$ to $10 \%$ ) may limit their effectiveness. This may be related to the eye's natural protective barriers; for example, the tear film may dilute the formulation and clear it rapidly through normal drainage channels before the drug can be absorbed (ie, a short residence time). ${ }^{13,14}$ In addition, chronic uninterrupted steroid use may lead to possible side effects, including cataract formation and elevation of intraocular pressure (IOP). ${ }^{15}$ The use of pulsatile dosing for corticosteroids may help minimize the safety related concerns; however, this approach does not address the limited ocular bioavailability. Therefore, alternative corticosteroid dosing techniques that enhance drug bioavailability in the eye, such as iontophoresis, may be a viable therapeutic option.

In ocular iontophoresis, a small applied current forms an electrical field at the ocular surface, which crosses into anterior and posterior segments. Under the influence of this field, the mobility of charged particles becomes enhanced. Placing drug molecules into the field may lead to substantially higher ocular drug concentrations than achieved with eyedrops. In 1943, the first ocular iontophoretic application was reported. ${ }^{16}$ Later, Maurice and colleagues advanced the use of iontophoresis as a means to enhance drug delivery to ocular tissues. ${ }^{17,18}$ More recently, Lam et a ${ }^{19}$ showed that iontophoresis-delivered vitreal dexamethasone was 3500-and 700-fold higher than either topical instillation or subconjunctival injection, respectively. This enhanced delivery can also prolong the duration of drug action, as demonstrated in a study of iodide iontophoresis in which extended drug exposure was demonstrated compared to topical iodide application without current. ${ }^{20}$

In order to develop an optimal drug product for use with ocular iontophoresis, physicochemical properties of candidate compounds must be considered, and special attention must be paid to formulation optimization, including drug substance concentration, excipient content, and osmolality. ${ }^{21}$

Dexamethasone and dexamethasone phosphate have demonstrated long-term safety and efficacy in humans, and have well-characterized safety and efficacy profiles for ophthalmic indications. Dexamethasone phosphate is a prodrug of dexamethasone, and readily converts to dexamethasone in vivo. Dexamethasone lacks a charged group and has limited aqueous solubility $(0.1 \mathrm{mg} / \mathrm{mL}){ }^{22}$ In contrast, dexamethasone phosphate possesses 2 acidic protons that allow the production of highly water soluble formulations of a charged species within the $\mathrm{pH}$ range of 5.5 to 7.4; therefore, it is a suitable candidate for iontophoresis. ${ }^{19}$

A novel ocular iontophoresis platform, the EyeGate ${ }^{\circledR}$ II system (Eyegate Pharmaceuticals, Inc, Waltham, MA), has been designed to deliver drugs through the conjunctiva and sclera. ${ }^{23}$ Preclinical studies guided the development of a $40 \mathrm{mg} / \mathrm{mL}$ dexamethasone phosphate solution (EGP-437, Eyegate Pharmaceuticals, Inc) for specific use with the Eyegate ${ }^{\circledR}$ II device. In rabbits, iontophoresis of EGP-437 delivered significantly higher $(\sim 100+$ fold $)$ aqueous humor drug concentration levels than that achieved with either topical or intravenous administration..$^{23}$ Furthermore, in a rabbit model of dry eye, a single iontophoresis treatment of $40 \mathrm{mg} / \mathrm{mL}$ dexamethasone phosphate solution led to full recovery within 2 days, as measured by corneal fluorescein staining and tear volume, using Schirmer's test; these effects were sustained through the 10-day duration of the study. ${ }^{24}$

The clinical study described herein explored the safety and efficacy of ocular iontophoresis of EGP-437 $(40 \mathrm{mg} / \mathrm{mL}$ dexamethasone phosphate solution) in dry eye patients. 


\section{Methods}

This single-center, double-masked, randomized, placebocontrolled phase II trial evaluated ocular iontophoresis of EGP-437 in patients with moderate to severe dry eye syndrome. Patients were recruited from an existing patient database or by using an Institutional Review Board-approved advertisement. General guidelines for dry eye diagnosis followed standards set at the international dry eye workshop. ${ }^{1}$ Enrollment was open to patients at least 12 years of age, who had a medical history of ongoing moderate to severe dry eye, reported the use or desire to use artificial tears in both eyes, and demonstrated best corrected visual acuities in both eyes of $0.5 \log$ MAR or better on the ETDRS chart.

Patients were excluded from study enrollment if they had known contraindications, allergy, or sensitivity to the test articles. They were also excluded if they were currently pregnant, nursing, or planning a pregnancy; had any medical conditions that in the investigator's opinion could impede the iontophoresis application (eg, pacemakers) or study parameters, or that could put the patient's health at substantial risk. Pregnancy tests were administered to females of childbearing potential at visit 1 , and those patients were asked to use an acceptable contraceptive method for the duration of the study. Follow-up pregnancy tests were administered at visit 7. Patients who had used certain medications (other investigative drugs, ocular drying medications, ocular steroids, Restasis ${ }^{\circledR}$ ) within the previous 4 weeks were also excluded. Contact lens wearers and those who had undergone LASIK surgery within the previous 12 months were also excluded.

The study used the Controlled Adverse Environment (CAE) mode ${ }^{25}$ developed by Ora, Inc, Andover, MA to assess the signs and symptoms of dry eye. This model is designed to emulate extreme environmental conditions (eg, low humidity, high temperature, visual tasking) that contribute to drying of the ocular surface. The CAE system exacerbates the signs and symptoms of dry eye in a reproducible reversible manner, ${ }^{25-28}$ and correlates with murine models of dry eye disease. ${ }^{29}$

\section{Study design}

This exploratory study was designed to evaluate effects among different clinical scenarios: treatment, the effects of iontophoresis-delivered dexamethasone phosphate following CAE-induced dry eye signs and symptoms; prevention, the effects of iontophoresis-delivered dexamethasone phosphate prior to CAE exposure; recovery, the effects of iontophoresisdelivered dexamethasone phosphate on recovery toward baseline at follow-up visits ( 24 hours and 7 days after CAE); and "environmental" (periods of time not directly influenced by the CAE), the ability of iontophoresis-delivered dexamethasone phosphate to improve dry eye signs and symptoms over the entire 3-week study period.

The study included 7 visits over 3 weeks: 3 visits (visits 1, 3, and 5), in which patients were exposed to the CAE for 90 minutes, and 4 follow-up visits (visits 2, 4, 6, and 7) (Figure 1). At visit 1 (day $-7 \pm 2$ ), patients provided written informed consent prior to any study procedures, and clinical staff collected medical and medication history data. Prior to and after CAE exposure, patients completed symptom questionnaires, had their blink rates captured using an infrared camera mounted on a headset, underwent ophthalmic exams including slit lamp biomicroscopy, and conjunctival redness grading based on the Ora 5-point scale ( $0=$ none to $4=$ severe $)$. Ocular surface staining using the $\mathrm{Ora}^{28}$ regions was assessed on a 5 -point scale $(0=$ none to $4=$ confluent). Fluorescein and lissamine green stains were used - fluorescein to assess the cornea and lissamine green to evaluate the conjunctiva. Tear film break-up time (TFBUT) data were also captured. Ocular Protection Index (OPI) values were calculated by dividing the TFBUT by the mean interblink interval (derived from blink rate). ${ }^{30} \mathrm{~A}$ complete enumeration of procedures and assessments is provided in Table 1.

During CAE exposure, patients reported ocular discomfort scores on a 5 -point scale $(0=$ none to $4=$ severe $)$ every 5 minutes..$^{28}$ After completing CAE exposure, investigators assessed corneal sensitivity using a Cochet-Bonnet aesthesiometer. In order to ensure that the study sample included patients with significant disease and a dynamic range of relevant signs and symptoms, patients were screened for demonstrated baseline signs and symptoms of dry eye prior to $\mathrm{CAE}$ exposure (corneal staining $>1$ and presence of conjunctival redness) and a CAE-induced response such as increases in ocular discomfort scores and fluorescein staining in at least 1 eye. This value for corneal staining corresponds to occasional fluorescein deposition. Qualifying patients received a diary in which to record their symptoms 3 times daily for the duration of the study.

Visits 1 and 3 procedures were similar. Patients who continued to meet qualification criteria after visit 3 (day 0 ) CAE exposure were randomized into the study according to a predefined randomization code generated by an independent biostatistician, and received ocular iontophoresis of either: dexamethasone phosphate $40 \mathrm{mg} / \mathrm{mL}, 7.5 \mathrm{~mA}-\mathrm{min}$ at $2.5 \mathrm{~mA}$ (DP 7.5); dexamethasone phosphate $40 \mathrm{mg} / \mathrm{mL}$, $10.5 \mathrm{~mA}$-min at $3.5 \mathrm{~mA}$ (DP 10.5); or sodium citrate buffer 


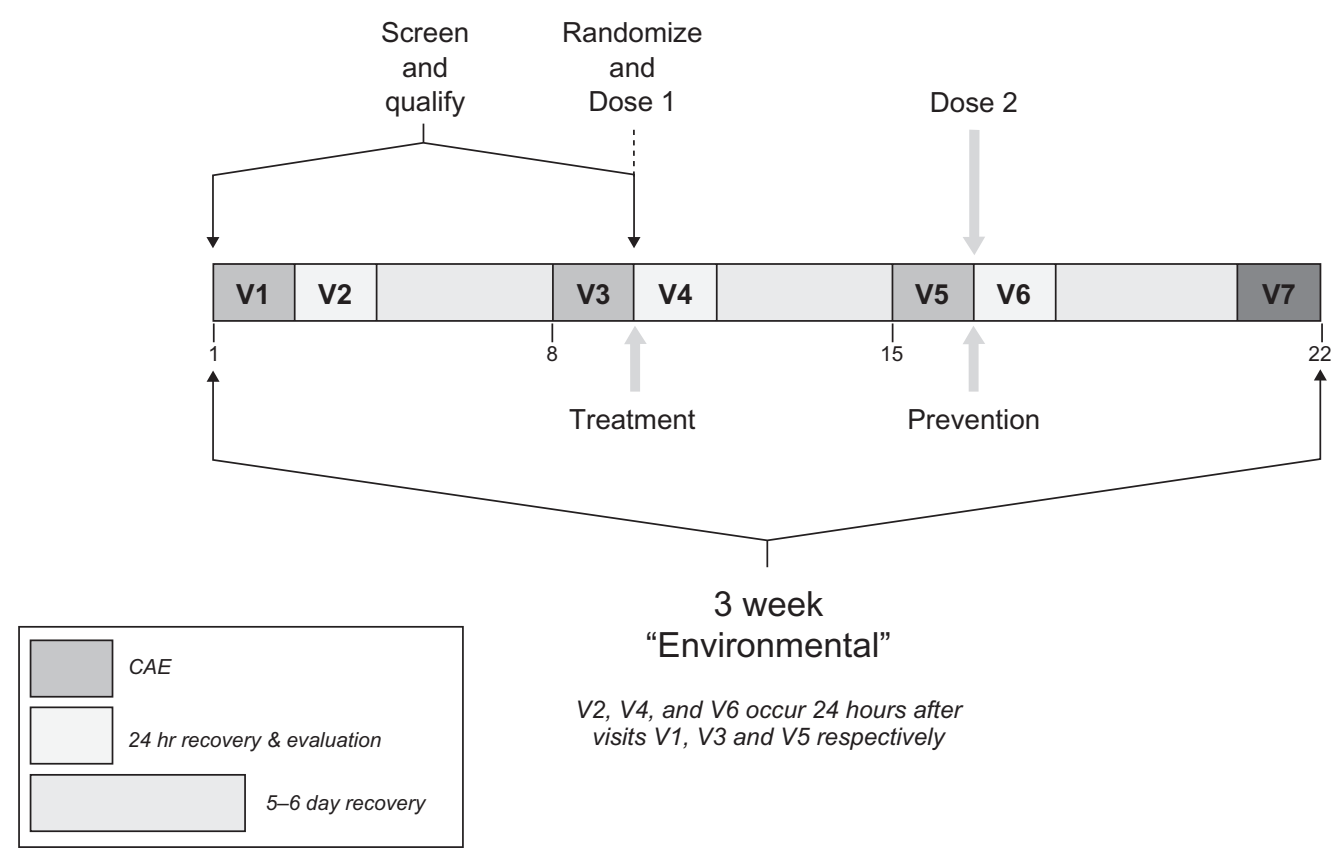

Figure I Study visit schedule. Diagram represents the overall study timeline, delineating the temporal relationship between screening visits, Controlled Adverse Environment (CAE) and drug dosing sessions, and the recovery period.

solution $100 \mathrm{mM}, 10.5 \mathrm{~mA}-\mathrm{min}$ at $3.5 \mathrm{~mA}$ (placebo). An unmasked technician identified the proper treatment group according to the randomization scheme, and then loaded the appropriate drug product from a standard vial into the foam reservoir of the ocular applicator. At least 1 hour after qualifying patients exited the CAE, patients were treated. Prior to placing the ocular iontophoresis applicator on the eye, the masked investigator instilled a topical anesthetic. The unmasked technician programmed the appropriate dose into the iontophoresis generator, concealed the device from the investigator, and then initiated treatment. The unmasked technician performed no other study procedures. The treatment process was then repeated for the other eye (Figure 2). Visit 5 procedures were the same as those at visit 3 , but the visit 5 iontophoresis treatments were performed prior to CAE exposure.

At the follow-up visits (visit 2 [day $-7+24 \pm 3$ hours], visit 4 [day $0+24 \pm 3$ hours], visit 6 [day $7+24 \pm 3$ hours], and visit 7 [day $14 \pm 2$ ]), medical and medication histories were updated, and patients underwent all visit 1 study procedures except for CAE exposure and post-CAE exams and questionnaires. Patients were asked to complete an additional ocular discomfort assessment at visits 4, 5, 6, and 7, as well as an overall global rating for the treatments at visit 7. Adverse events (AEs) that were elicited from patients or observed by investigators for the duration of the study were recorded and assessed for severity and possible cause.

\section{Statistical methods}

The sample size determination of 30 in each DP group and 20 in the placebo group was based on a family-wise type I error of 0.05 . Assuming common standard deviation of 1.2 units for corneal fluorescein staining typical of CAE studies, the sample size determination had approximately $80 \%$ power to identify true mean difference in corneal staining of 1.0 unit. Likewise, assuming common standard deviations of 1.0 unit for ocular discomfort in the CAE, the sample size described above was estimated to have $90 \%$ power to identify true 1.0-unit mean difference in ocular discomfort.

Every randomized patient, for whom there was any evidence that study medication was used, and for whom follow-up safety data were available, was considered evaluable for safety purposes, comprising the safety population. The intent-to-treat (ITT) population included all randomized patients. The per-protocol (PP) population excluded ITT patients (prior to locking the database) with any major protocol deviations that could impact the efficacy analysis.

Primary efficacy analyses (DP dose groups compared with placebo, corneal fluorescein staining post-CAE at visit 5 and ocular discomfort during CAE exposure at visit 5) were performed on the ITT population using the worst eligible (WE) eye. The technique of last observation carried forward (LOCF) was used in cases of missing efficacy data variables. Corneal fluorescein staining results were analyzed using a $t$-test and a Wilcoxon rank sum 


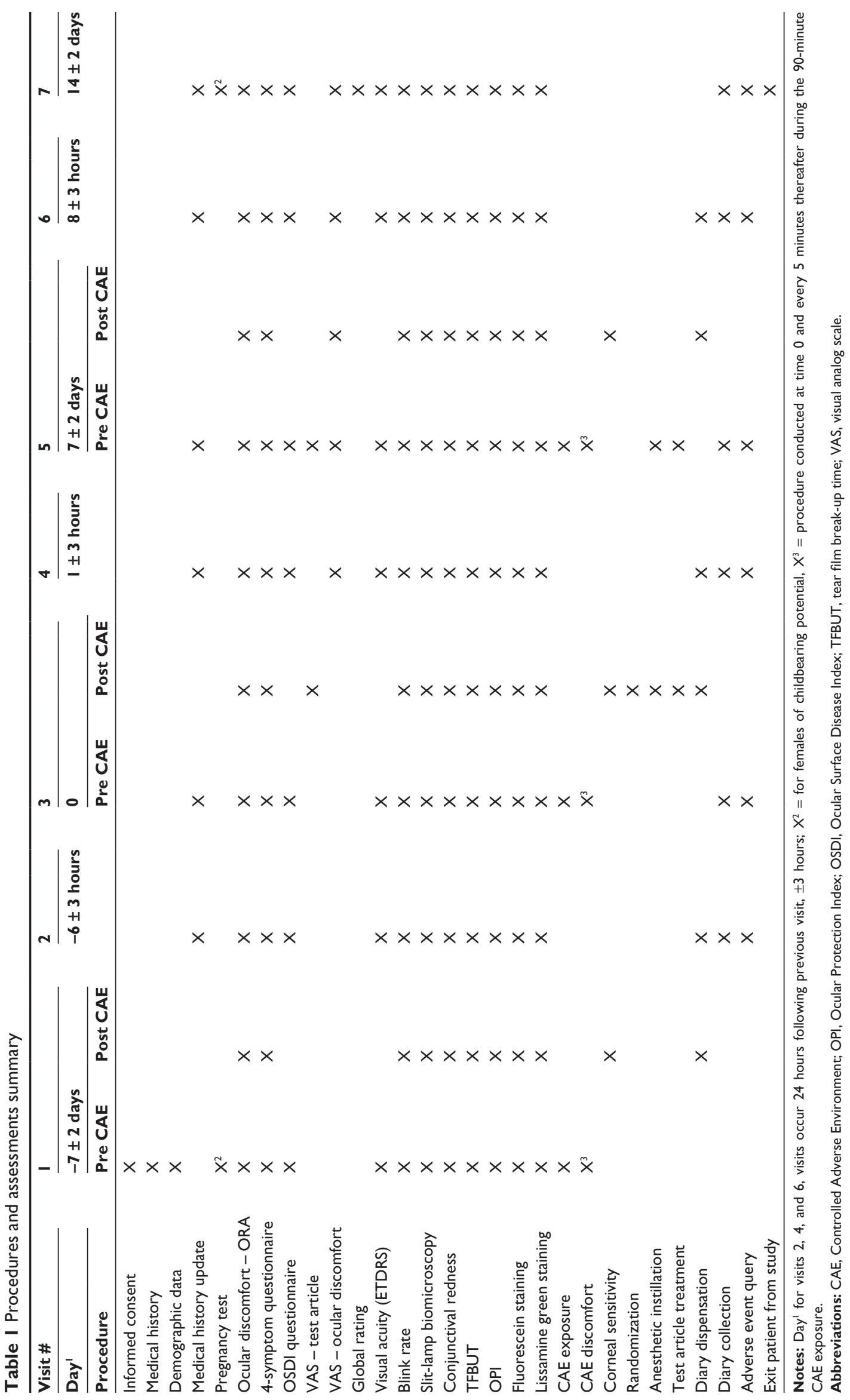




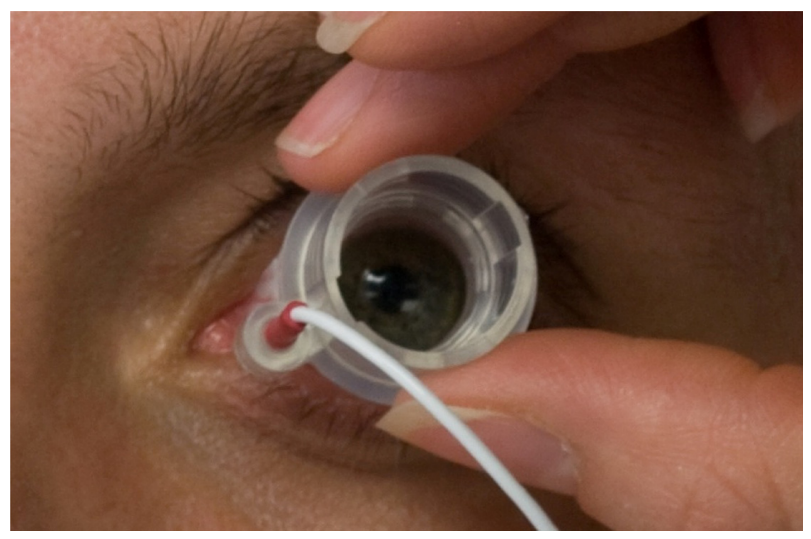

Figure 2 Ocular iontophoresis application. The photo shows the iontophoretic applicator placement on the eye.

test during the treatment period (pre- and post-CAE), and an analysis of covariance (ANCOVA) model was used to calculate least square means (LS means) and $P$ values. Ocular discomfort data during CAE exposure were used to calculate the area under the curve (AUC) for each eye using the trapezoidal rule. ${ }^{31}$ Statistical hypothesis testing was primarily performed for the worse eye by using a linear model (ANOVA) to assess treatment group differences with AUC as the response. Secondarily, statistical hypothesis testing was also performed by an ANCOVA model with time, treatment effects, and the interactions carried out across all time points accounting for repeated measures per patient.

For secondary efficacy variables, all pair-wise comparisons for each quantitative variable were performed using both a $t$-test and a Wilcoxon rank sum test during the treatment period; an ANCOVA model was used to calculate LS Means and $P$ values. A Chi-square test, or Fisher's exact test in the case of expected counts $<5$, was used for the discrete or binary variables without stratification for all pair-wise comparisons. Additional analyses for the secondary efficacy variables were performed using the same statistical models, on the following analysis populations: ITT-observed data only (ODO)-WE, ITT-ODO-all eligible eyes (AEE), PP population-WE, and PP population-AEE. All treatment effects were assessed at a significance level of $5 \%$. All safety analyses were performed on the safety population. AEs were coded using the MedDRA dictionary (version 11.0) and classified by system organ class and preferred term. A series of post-hoc analyses were carried out to explore areas where the primary or secondary endpoints demonstrated statistically significant findings or indicated trends of interest. For consistency, the secondary efficacy and post-hoc findings are discussed for the PP-WE analysis population.

\section{Results \\ Demographics}

A total of 105 patients (mean age: 53 years, range 18 to 84; $32 \%$ male; $89 \%$ Caucasian) were randomized into three treatment groups comprising the ITT population. There were no significant differences between groups for age or male/ female ratios. One hundred and three patients received study treatments via iontophoresis, comprising the safety population. Of the 105 randomized patients, 2 withdrew from the study prior to receiving treatment. The PP population consisted of the 83 patients who completed the study with no major protocol violations. The patient disposition is shown in Figure 3. Fourteen patients did not complete the study for various reasons, including two that received the wrong treatment. No patient was discontinued from the study as a result of a protocol deviation; however, six patients were excluded from the PP population for the following reasons: completed visit 5 out-of-window $(n=4)$, took exclusionary medication $(n=1)$, and received the wrong treatment via technician error $(n=1)$. Patients who received the wrong treatment were included in the ITT population as treated.

\section{Signs}

There were no statistically significant differences among the treatment groups for the primary sign endpoint of corneal staining post-CAE at visit 5 in the ITT-LOCF-WE analysis population. However, the DP 7.5 treatment group demonstrated less lissamine green corneal staining than the placebo group pre- to post-CAE at visit 5 in the superior region (PP-WE; $P=0.017, t$-test; $P=0.039$, Wilcoxon). Statistically significant improvements in TFBUT were observed for DP 7.5 treatment relative to placebo at visit 5 pre- and post-CAE (PP-WE; $P=0.034,0.049$, respectively, $t$-test) and at visit 7 (PP-WE; $P=0.042, t$-test). Significant improvements in OPI were observed for DP 7.5 treatment relative to placebo at visit 5 post-CAE (PP-WE; $P=0.048$, Chi-square test). While the DP 10.5 treatment group values displayed improvements compared with placebo for all of these criteria, none reached the level of statistical significance $(P<0.05)$. A listing of statistically significant findings is shown in Table 2.

At visit 7, differences between the DP 7.5 treatment and placebo groups were documented, including the TFBUT as described above and a trend toward improved corneal fluorescein staining (inferior region of the Ora scale in the ITT-LOCF-WE, $P=0.086$, two-sample t-test and $P=0.092$, Wilcoxon). When comparing endpoints across the entire study's duration (between visit 1 baseline and visit 7 


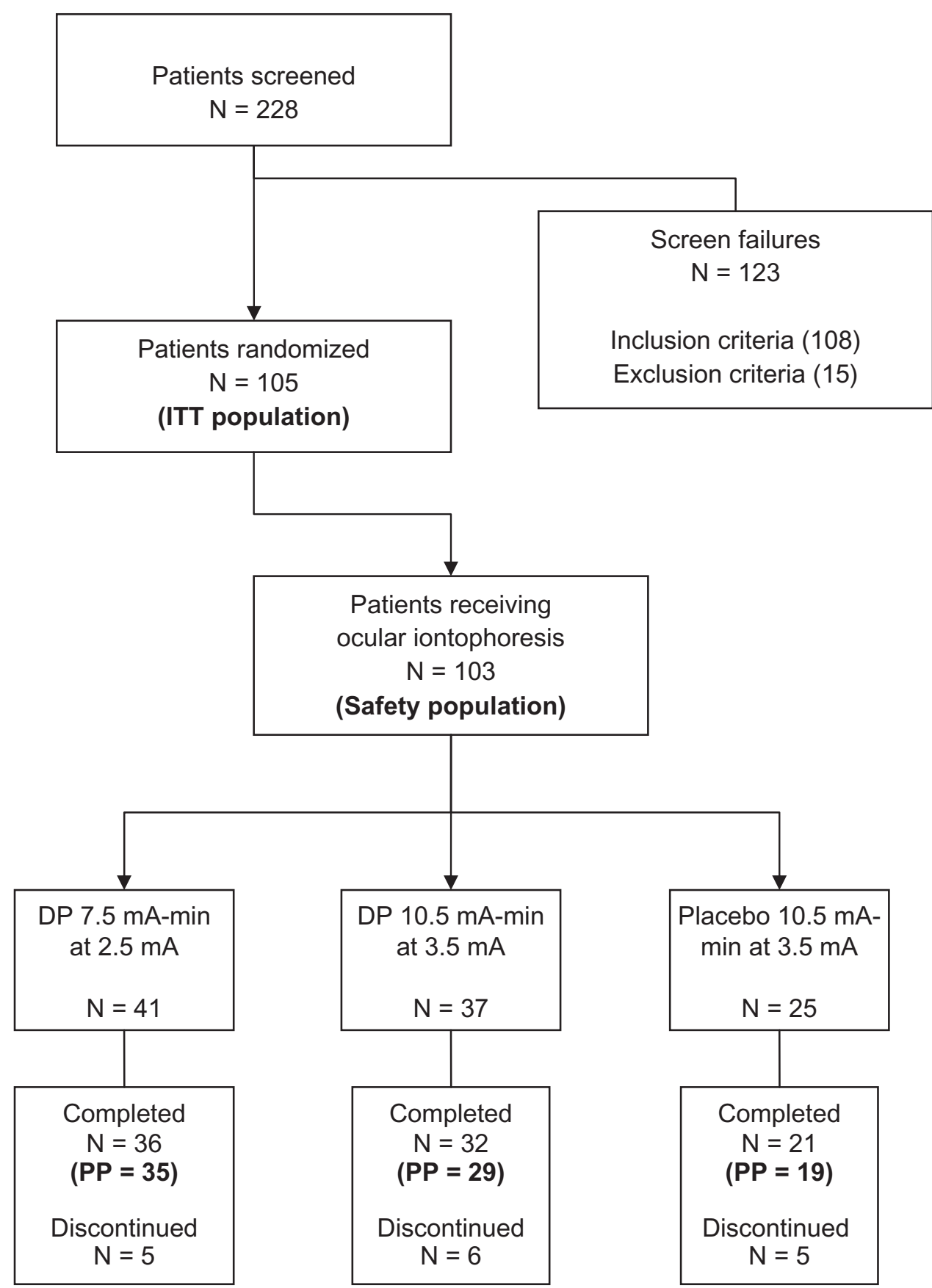

Figure 3 Patient disposition. The flow chart depicts the distribution of patients throughout the study as well as inclusion/exclusion numbers, study populations, and treatment arms.

Abbreviations: DP, dexamethasone phosphate; ITT, intention to treat; PP, per protocol.

study exit), a statistically significant decrease in fluorescein staining in the inferior region of the Ora scale was revealed for the DP 7.5 treatment group over placebo $(0.51, P=0.038$, Wilcoxon, Figure 4).

\section{Symptoms}

The differences in mean ocular discomfort scores between both DP groups and placebo during CAE exposure at visit 5, the primary endpoint for symptoms, were not statistically significant; however, the differences in the mean ocular discomfort scores for DP 7.5 versus placebo at several discrete time points during the visit $5 \mathrm{CAE}$ exposure were statistically significant (PP-WE, Figure 5). In order to determine if the patients reporting lower ocular discomfort scores during the visit $5 \mathrm{CAE}$ experienced improvements in any relevant dry eye signs, two subgroups of patients 
Table 2 Secondary endpoints: statistically significant results compared with placebo shown in bold ( $p<0.05)$.

\begin{tabular}{|c|c|c|c|c|c|c|}
\hline Endpoint & Analysis population & Time point & & DP 7.5 & DP 10.5 & Placebo \\
\hline \multirow{3}{*}{$\begin{array}{l}\text { Lissamine green staining } \\
\text { (superior region) }\end{array}$} & PP-WE & Visit 5: change pre & Mean (SD) & $\mathrm{I} .60(0.88 \mathrm{I})$ & I.78 $(0.84 \mathrm{I})$ & $2.11(0.614)$ \\
\hline & & to post-CAE & $P$ value ${ }^{\prime}$ & 0.0173 & 0.1242 & - \\
\hline & & & $P$ value $^{2}$ & 0.0385 & 0.1965 & - \\
\hline \multirow[t]{6}{*}{ TFBUT } & PP-WE & Visit 5, pre-CAE & Mean (SD) & $2.25(0.854)$ & $2.32(1.193)$ & $\mathrm{I} .82(0.57 \mathrm{I})$ \\
\hline & & & $P$ value ${ }^{\prime}$ & 0.0339 & 0.0599 & - \\
\hline & & Visit 5, post-CAE & Mean (SD) & $2.04(0.750)$ & $2.09(0.820)$ & $1.68(0.528)$ \\
\hline & & & $P$ value & 0.0488 & 0.0403 & - \\
\hline & & Visit 7 & Mean (SD) & $2.73(1.314)$ & $2.39(1.314)$ & $1.95(1.119)$ \\
\hline & & & $P$ value & 0.0417 & 0.2198 & - \\
\hline \multirow[t]{3}{*}{ OPI } & PP-WE & Visit 5, post-CAE & $N(\%) \geq I$ & 19 (54.3\%) & II (37.9\%) & $5(26.3 \%)$ \\
\hline & & & $N(\%)<1$ & $16(45.7 \%)$ & $18(62.1 \%)$ & I4 (73.7\%) \\
\hline & & & $P$ value $^{3}$ & 0.0482 & 0.4038 & - \\
\hline \multirow[t]{6}{*}{ Ocular discomfort } & PP-WE & Visit 4 & Mean (SD) & $1.29(0.957)$ & $\mathrm{I} .34(\mathrm{I} .26 \mathrm{I})$ & $1.79(0.787)$ \\
\hline & & & $P$ value ${ }^{\prime}$ & 0.0437 & 0.1396 & - \\
\hline & & & $P$ value ${ }^{2}$ & 0.0320 & 0.0971 & - \\
\hline & & Visit 6 & Mean (SD) & $1.03(0.985)$ & $1.24(1.091)$ & $1.84(0.898)$ \\
\hline & & & $P$ value ${ }^{\prime}$ & 0.0038 & 0.0436 & - \\
\hline & & & $P$ value $^{2}$ & 0.0032 & 0.0360 & - \\
\hline Visual analog scale (VAS) & PP & Visit 5, pre-CAE & Mean (SD) & $1.86(0.772)$ & $\mathrm{I} .66(0.936)$ & $2.16(0.688)$ \\
\hline \multirow[t]{2}{*}{ (ocular discomfort) } & & & $P$ value ${ }^{\prime}$ & 0.1498 & 0.0377 & - \\
\hline & & & $P$ value $^{2}$ & 0.1746 & 0.0406 & - \\
\hline
\end{tabular}

Notes: 'Two-sample $t$-test; ${ }^{2}$ Wilcoxon rank-sum test; ${ }^{3}$ Chi square test; All $P$ values calculated relative to placebo.

Abbreviations: AEE, all eligible eyes; CAE, Controlled Adverse Environment; ODO, observed data only; PP, per protocol; WE, worst eye.

were evaluated: those demonstrating ocular discomfort scores of $<3$ and those demonstrating ocular discomfort scores $<4$ at all time points between 50 and 90 minutes during visit $5 \mathrm{CAE}$ exposure. For patients in the subgroup scoring $<4$ at all time points between 50 and 90 minutes during visit $5 \mathrm{CAE}$ exposure, the visit 6 and 7 data demonstrated significantly longer mean TFBUTs for both

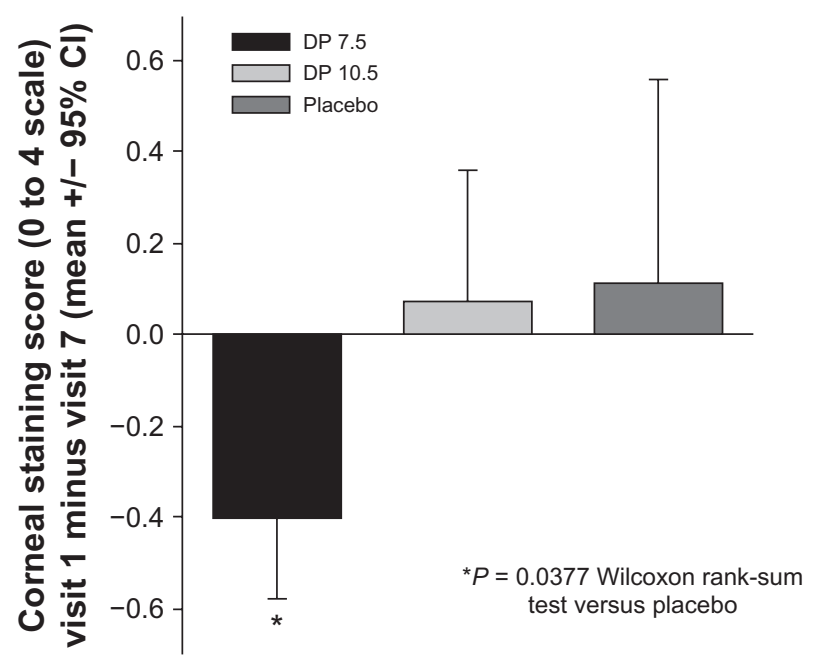

Figure 4 Corneal staining is decreased by iontophoretic dexamethasone phosphate. The mean change in corneal fluorescein staining (inferior region) between visit I (baseline) to visit 7 for each treatment group. Over this time frame, the DP 7.5 treatment group showed a statistically significant decrease in staining compared with the placebo treatment group.

Abbreviation: DP, dexamethasone phosphate. active treatment groups compared with the placebo group (Table 3).

The ocular discomfort scores at visits 4 and 6 decreased by about 1 unit on a scale of 0 to 4 and were statistically significant in the DP 7.5 treatment group versus placebo ( $P=0.032$ and $P=0.0032$, respectively, Wilcoxon).

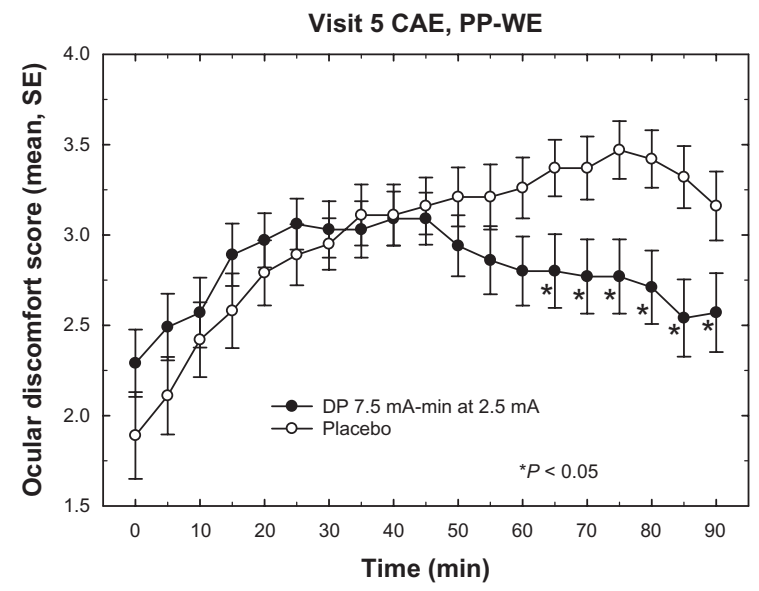

Figure 5 Mean ocular discomfort scores during CAE exposure at visit 5. The plot depicts the mean discomfort scores for the placebo and DP 7.5 treatment groups during the course of the visit 5 CAE session. The DP 10.5 group, which was not significantly different from placebo, is omitted for clarity. Data included in the mean value calculation were from the PP-WE population of each group. The DP 7.5 group mean values are significantly lower $(P<0.05)$ than placebo for all times $>60$ minutes. Error bars represent standard error of the mean.

Abbreviations: CAE, Controlled Adverse Environment; DP, dexamethasone phosphate; PP-WE, per protocol-worst eye. 
Table 3 TFBUT in ocular discomfort subgroups. Values in bold are statistically significant $(p<0.05)$

\begin{tabular}{|c|c|c|c|c|}
\hline & & DP 7.5 & DP 10.5 & Placebo \\
\hline \multicolumn{2}{|c|}{ Subgroup scoring $<3$} & $N=9$ & $N=5$ & $N=2$ \\
\hline \multirow[t]{3}{*}{ Visit 6} & Mean (SD) & $1.99(0.715)$ & $2.13(1.314)$ & $1.33(0.113)$ \\
\hline & Range & $(1.4-3.7)$ & $(I . I-4.4)$ & $(1.3-1.4)$ \\
\hline & $P$ value & $0.028 I$ & 0.2484 & - \\
\hline \multirow[t]{3}{*}{ Visit 7} & Mean (SD) & $2.79(2.284)$ & $2.39(0.457)$ & $1.26(0.233)$ \\
\hline & Range & $(1.3-8.5)$ & $(1.6-2.8)$ & $(I . I-I .4)$ \\
\hline & $P$ value & 0.0822 & 0.0123 & - \\
\hline \multicolumn{2}{|c|}{ Subgroup scoring $<4$} & $N=20$ & $N=16$ & $N=8$ \\
\hline \multirow[t]{3}{*}{ Visit 6} & Mean (SD) & $2.65(1.98 I)$ & $2.42(1.275)$ & I.44 (0.257) \\
\hline & Range & $(I .1-9.2)$ & $(I . I-5.2)$ & $(1.1-1.8)$ \\
\hline & $P$ value & 0.0144 & 0.0088 & - \\
\hline \multirow[t]{3}{*}{ Visit 7} & Mean (SD) & $2.60(1.693)$ & $2.76(1.553)$ & $1.68(0.623)$ \\
\hline & Range & $(1.0-8.5)$ & $(1.2-6.6)$ & $(0.9-2.6)$ \\
\hline & $P$ value & 0.0437 & 0.0239 & - \\
\hline
\end{tabular}

Abbreviations: DP, dexamethasone phosphate; TFBUT, tear film break-up time.

\section{Safety}

Over the course of the study, $90(87 \%)$ of the 103 patients in the safety population experienced at least $1 \mathrm{AE}$, including 37 patients in the DP 7.5 treatment group, 34 patients in the DP 10.5 treatment group, and 19 patients in the placebo group. Ocular AE data are summarized in Table 4. Of the 254 treatment-emergent AEs [(TEAEs) AEs arising after patients received first treatment], 199 (78\%) were ocular: 77 in the DP 7.5 treatment group, 82 in the DP 10.5 treatment group, and 40 in the placebo group. The most commonly reported ocular TEAEs were hyperemia and keratitis. No adverse effects on visual acuity were observed. Ten patients (9.7\%) were withdrawn from the study due to AEs; of these, 3 were in the placebo treatment group (12\%), 4 were in the DP 7.5 treatment group (9.8\%), and 3 were in the DP 10.5 treatment group $(8.1 \%)$. The majority of all treatment-related ocular AEs resolved without sequelae within 24 hours. No severe AEs were observed.

\section{Discussion}

Currently, eyedrops are the main treatment regimen for dry eye patients. In this exploratory study, iontophoretically delivered dexamethasone phosphate (EGP-437) demonstrated significant improvements in a variety of signs and symptoms of dry eye relative to placebo. Despite missing the primary endpoints at visit 5 in the ITT population, statistically significant improvements were demonstrated in each of the 4 testing scenarios (treatment, prevention, recovery, and "environmental") for the PP population.

Table 4 Subject-reported ocular TEAEs. The totals for each group, along with the number of subjects with multiple AEs, are in bold for emphasis.

\begin{tabular}{|c|c|c|c|}
\hline & DP $7.5(\mathrm{~N}=41)$ & DP I0.5 ( $N=37)$ & Placebo $(\mathbf{N}=\mathbf{2 5})$ \\
\hline Total number of ocular AEs & 77 & 82 & 40 \\
\hline Subjects with $\geq I$ ocular $A E^{\prime}$ & $36(87.8 \%)$ & 31 (83.8\%) & $16(64.0 \%)$ \\
\hline Eye disorders' & 27 (65.9\%) & $27(73.0 \%)$ & $15(60.0 \%)$ \\
\hline Ocular hyperemia & 16, $3(39.0 \%)$ & I8, 2 (48.6\%) & II, I (44.0\%) \\
\hline Keratitis & $6,3(14.6 \%)$ & $6,4(16.2 \%)$ & I, 0 (4.0\%) \\
\hline Ocular discomfort & $4,2(9.8 \%)$ & $6,1(16.2 \%)$ & $2,0(8.0 \%)$ \\
\hline Conjunctival edema & 4,0 (9.8\%) & $3,1(8.1 \%)$ & $2,0(8.0 \%)$ \\
\hline Vision blurred & $4,0(9.8 \%)$ & $2,0(5.4 \%)$ & I, 0 (4.0\%) \\
\hline Eye irritation & I, 0 (2.4\%) & $3,1(8.1 \%)$ & $2,0(8.0 \%)$ \\
\hline Eye pain & 3,2 (7.3\%) & $\mathrm{I}, \mathrm{I}(2.7 \%)$ & $2,0(8.0 \%)$ \\
\hline Foreign body sensation in eyes & $2,1(4.9 \%)$ & $3,1(8.1 \%)$ & $\mathrm{I}, 0$ (4.0\%) \\
\hline Photopsia & $2,0(4.9 \%)$ & $2,0(5.4 \%)$ & I, 0 (4.0\%) \\
\hline Photophobia & 0 & $3,1(8.1 \%)$ & I, 0 (4.0\%) \\
\hline Punctate keratitis & $2,0(4.9 \%)$ & I, $0(2.7 \%)$ & 0 \\
\hline Dry eye & $\mathrm{I}, 0$ (2.4\%) & I, 0 (2.7\%) & 0 \\
\hline Eye pruritis & $\mathrm{I}, \mathrm{I}(2.4 \%)$ & 0 & I, I (4.0\%) \\
\hline Abnormal sensation in eye & $\mathrm{I}, \mathrm{I}(2.4 \%)$ & 0 & 0 \\
\hline Corneal deposits & 0 & I, 0 (2.7\%) & 0 \\
\hline Erythema of eyelid & $\mathrm{I}, 0$ (2.4\%) & 0 & 0 \\
\hline Eyelid margin crusting & 0 & 0 & I, 0 (4.0\%) \\
\hline Lacrimation increased & 0 & I, I (2.7\%) & 0 \\
\hline Lenticular opacities & I, 0 (2.4\%) & 0 & 0 \\
\hline Vitreous detachment & 0 & I, 0 (2.7\%) & 0 \\
\hline Vitreous floaters & I, 0 (2.4\%) & 0 & 0 \\
\hline
\end{tabular}

Notes: 'Table entries [n, $\mathrm{m}(\%)]$ represent the total number $(\mathrm{n})$ of subjects reporting I or more treatment-emergent ocular AEs. The number of those classified as moderate $(\mathrm{m})$, and the corresponding percentage of total subjects in that treatment group.

Abbreviations: AE, adverse event; TEAE, treatment-emergent adverse event; DP, dexamethasone phosphate. 
The decreased ocular discomfort reported during the visit 5 CAE between the 60- and 90-minute timepoints (within 2 hours of the second treatment), observed 24 hours (recovery) following CAE exposure at visits 4 (treatment) and 6 (prevention), pre- to post-CAE comparison of lissamine green corneal staining at visit 5 (prevention), TFBUT post-CAE at visit 5 (prevention), and OPI post-CAE at visits 5 (prevention) and 6 (prevention and treatment) suggest a rapid onset of action for iontophoresis-delivered EGP-437.

Fluorescein staining in the inferior region (Ora scale) is recognized as an important sign of dry eye disease, because this area represents a region specifically vulnerable to exacerbation by stress conditions, ${ }^{25}$ including those presented in the CAE model. The DP 7.5 treatment improved the inferior region (Ora scale) fluorescein staining observed from visit 1 baseline to visit 7 by 0.51 units relative to placebo ("environmental") demonstrating that the 2 iontophoresis treatments over the 3 weeks may aid healing in this region. These findings, in conjunction with the increased TFBUT measured at visit 5 pre-CAE (a week after the first iontophoresis treatment), and visit 7 (a week after the second iontophoresis treatment), as well as the ocular discomfort score at visit 5 pre-CAE, suggest that iontophoresis-delivered EGP-437 may provide beneficial effects for dry eye patients without the need for daily dosing.

Since multiple statistically significant observations were made across a variety of visits and endpoints, it is likely that the effects are treatment-related. Based on all endpoints analyzed, it appears that the lower dose, DP 7.5 is more effective than the higher dose, DP 10.5. One possible explanation for the lower efficacy of the higher dose may be a difference in ocular drug distribution. It is possible that the higher current dose may drive the drug more deeply within the globe rather than delivering more drug to the ocular surface and anterior segment, as revealed in rabbit biodistribution studies. ${ }^{23}$ In response to this observation, future research will likely focus on treatment effects of dexamethasone phosphate $40 \mathrm{mg} / \mathrm{mL}$ at lower iontophoretic doses.

Although a majority of patients experienced some discomfort related to the iontophoresis procedures, most AEs were mild and there were no severe events. Most events were associated with the mild discomfort of having the device applied to the eye or with the sensations, for example, tingling in the eye during treatment. More AEs occurred at visit 3 than at visit 5 . This may be attributed to the fact that patients at visit 3 received treatment post-CAE exposure, at the time in the study when their eyes were most dry and irritated versus visit 5 when patients received treatment preCAE. Previous work with iontophoresis has shown similar treatment-related hyperemia that diminished after multiple iontophoretic applications..$^{20,32}$

The AEs of corneal staining in the trial described herein may have been a result of enhanced ocular surface vulnerability related to the CAE and/or to the external environment while patients received treatment with their eyes open for 3 minutes. Additional research is required to confirm this. Furthermore, electroencephalogram, electrocardiogram, electroretinogram, and IOP safety data obtained in an earlier healthy volunteer study utilizing this delivery system demonstrated no substantial risk of abnormalities. ${ }^{33}$

In another clinical study $(\mathrm{n}=40)$, following a single iontophoretic treatment with EGP-437, mean IOP remained relatively stable and within normal range in patients whose IOPs were monitored weekly over 28 days after treatment. ${ }^{34}$

If the drug exposure profiles in humans are similar to those measured in animals, ${ }^{23}$ then iontophoretic treatment with dexamethasone phosphate may convey similar safety advantages to those seen with therapeutic approaches using tapered or "pulse" systemic steroids relative to chronic steroid dosing.

EGP-437 treatment via the EyeGate ${ }^{\circledR}$ II system demonstrated positive effects on patients' dry eye signs and symptoms in the PP population and may represent a potential alternative dosing approach for corticosteroids in dry eye patients. However, the specific duration of action and dosing rationale still need to be established through additional studies. The study sample included a broad range of dry eye patients, and was not limited to those with low tear production; therefore, EGP-437 has the potential to be efficacious in a larger proportion of patients than Restasis ${ }^{\circledR}$. Further clinical work is required.

\section{Acknowledgments}

The authors thank Kathryn Kennedy, MSE of Statistics and Data Corporation, Tempe, AZ for statistical assistance; Morton Goldberg, MD, member of the Board of Directors, Eyegate Pharmaceuticals, Inc, Waltham, MA for helpful discussion and extensive review of the manuscript.

\section{Disclosure}

Financial support: The study was sponsored by Eyegate Pharmaceuticals, Inc. The sponsor participated in the design 
of the study, data analysis, and interpretation of the data, preparation, review and approval of the manuscript.

MAP, AC, and SF are employees and equity owners of Eyegate Pharmaceuticals, Inc. DW and GWO are employees of Ora, Inc, which received fees for conducting the study; and GT is a consultant of Ora, Inc.

Statement of conformity about author information: Institutional Review Board approval was obtained prior to any study procedures; all study patients had understanding of the trial and provided signed informed consent in order to participate; the study was conducted in accordance with local, State and Federal laws and the 1989 Declaration of Helsinki at a single study site in Andover, MA; and the details of the study were registered on clinicaltrials.gov (NCT00765804).

\section{References}

1. The Definition and Classification of Dry Eye Disease: Report of the Definition and Classification Subcommittee of the International Dry Eye Workshop. Ocul Surf. 2007;5(2):75-92.

2. The Epidemiology of Dry Eye Disease: Report of the Epidemiology Subcommittee of the International Dry Eye Workshop. Ocul Surf. 2007; 5:93-107.

3. Management and therapy of dry eye disease: Report of the Management and Therapy Subcommittee of the International Dry Eye Workshop. Ocul Surf. 2007;5:163-178.

4. Restasis ${ }^{\circledR}$ [prescribing information]. Irvine, CA: Allergan, Inc; 2009.

5. Perry HD, Solomon R, Donnenfeld ED, et al. Valuation of topical cyclosporine for the treatment of dry eye disease. Arch Ophthalmol. 2008;126:1046-1050.

6. Walker PM, Lane KJ, Ousler GW 3rd, Abelson MB. Diurnal variation of visual function and the signs and symptoms of dry eye. Cornea. 2010; 29:607-612.

7. Pflugfelder SC. Antiinflammatory therapy for dry eye. Am J Ophthalmol. 2004; $137: 337-342$.

8. Luo L, Li DQ, Corrales RM, Pflugfelder SC. Hyperosmolar saline is a proinflammatory stress on the mouse ocular surface. Eye Contact Lens. 2005;31:186-193.

9. Luo L, Li DQ, Doshi A, et al. Experimental dry eye stimulates production of inflammatory cytokines and MMP-9 and activates MAPK signaling pathways on the ocular surface. Invest Ophthal Vis Sci. 2004;45:4293-4301.

10. Huang AJW. Immunosuppressive therapy for ocular surface disorders. In: Dry Eye and Ocular Surface Disorders. Pflugfelder SC, Beuerman RW, Stern ME, editors. New York, NY. Marcel Dekker, Inc. 2004.

11. Barnes PJ. Corticosteroid effects on cell signalling. Eur Respir J. 2006;27:413-426.

12. American Academy of Ophthalmology Cornea/External Disease Panel. Preferred Practice Pattern: Dry Eye Syndrome. San Francisco, CA: American Academy of Ophthalmology; 2008.

Clinical Ophthalmology

\section{Publish your work in this journal}

Clinical Ophthalmology is an international, peer-reviewed journal covering all subspecialties within ophthalmology. Key topics include: Optometry; Visual science; Pharmacology and drug therapy in eye diseases; Basic Sciences; Primary and Secondary eye care; Patient Safety and Quality of Care Improvements. This journal is indexed on Submit your manuscript here: http://www.dovepress.com/clinical-ophthalmology-journal
13. Chun DK, Shapiro A, Abelson MB. Ocular Pharmacokinetics. In: Principles and Practice of Ophthalmology, 3rd ed. Albert DM, Miller JW, editors. Toronto, ON. Elsevier, Inc; 2008.

14. Ghate D, Edelhauser HF. Ocular drug delivery. Expert Opin Drug Deliv. 2006;3:275-287.

15. Li J, Tripathi RC, Tripathi BJ. Drug-induced ocular disorders. Drug Safety. 2008;31:127-141.

16. Von Sallmann L. Iontophoretic introduction of atropine and scopolamine into the rabbit eye. Arch Ophthalmol. 1943;29:711-719.

17. Hughes L, Maurice DM. A fresh look at iontophoresis. Arch Ophthalmol. 1984;102:1825-1829.

18. Jones RF, Maurice DM. New methods of measuring the rate of aqueous flow in man with fluorescein. Exp Eye Res. 1966;5:208-220.

19. Lam TT, Edward DP, Zhu XA, Tso MO. Transscleral iontophoresis of dexamethasone. Arch Ophthalmol. 1989;107:1368-1371.

20. Horwath-Winter J, Schmut O, Haller-Schober EM, et al. Iodide iontophoresis as a treatment for dry eye syndrome. $\mathrm{Br} J$ Ophthalmol. 2005;89:40-44.

21. Kalia YN, Naik A, Garrison J, Guy RH. Iontophoretic drug delivery. Adv Drug Deliv Rev. 2004;56:619-658.

22. Budavari S. Dexamethasone. In: The Merck Index: An Encyclopedia of Chemicals, Drugs, and Biologicals,14th ed. O'Neil MJ, editor. Whitehouse Station, NJ. Merck and Co; 2006.

23. Güngör S, Delgado-Charro MB, Ruiz-Perez B, et al. Trans-scleral iontophoretic delivery of low molecular weight therapeutics. $J$ Controlled Release. 2010;147:225-231.

24. Ruiz-Perez B, Dowie T, Gee R, Isom P, Manzo M, Skokanova E. Transscleral delivery of a $12.4 \mathrm{kDa}$ protein by ocular iontophoresis. Invest Ophthalmol Vis Sci. 2008;49:1813.

25. Ousler GW III, Gomes PJ, Welch D, Abelson MB. Methodologies for the study of ocular surface disease. Ocul Surf. 2005;3:143-154.

26. Ousler GW III, Anderson RT, Osborn KE. The effect of senofilcon A contact lenses compared to habitual contact lenses on ocular discomfort during exposure to a controlled adverse environment. Curr Med Res Opin. 2008;24:335-341.

27. González-García MJ, González-Sáiz A, et al. Exposure to a controlled adverse environment impairs the ocular surface of patients with minimally symptomatic dry eye. Invest Ophthalmol Vis Sci. 2007;48:4026-4032.

28. Ousler GW III, Wilcox KA, Gupta G, Abelson MB. An evaluation of the ocular drying effects of 2 systemic antihistamines: loratadine and cetirizine hydrochloride. Ann Allergy Asthma Immunol. 2004;93:460-464.

29. Crampton HJ, Ousler GW, Pflugfelder S, et al. Correlation of the Controlled Adverse Environment (CAE) model with a murine model of experimental dry eye in assessing the ability of topical doxycycline to prevent corneal barrier disruption. Invest Ophthalmol Vis Sci. 2007;48:402.

30. Ousler GW III, Hagberg KW, Schindelar M, et al. The Ocular Protection Index. Cornea. 2008;27:509-513.

31. Atkinson KA. An Introduction to Numerical Analysis, 2nd ed. New York: John Wiley \& Sons; 1989.

32. Halhal M, Renard G, Courtois Y, et al. Iontophoresis: from the lab to the bed side. Exp Eye Res. 2004;78:751-757.

33. Cohen A, Patane MA, Reddy M, Assang C, From S. Clinical Experience with the EyeGate ${ }^{\circledR}$ II Delivery System (EGDS): Safety and Tolerability in Healthy Adult Volunteers. Invest Ophthalmol Vis Sci. 2011;52:3224

34. Patane M, Cohen A, Sugarman J, From S. Randomized, double-masked study of four iontophoresis dose levels of EGP-437 in non-infectious anterior segment uveitis subjects. Invest Ophthalmol Vis Sci. 2010;51:52-63.

\section{Dovepress}

PubMed Central and CAS, and is the official journal of The Society of Clinical Ophthalmology (SCO). The manuscript management system is completely online and includes a very quick and fair peer-review system, which is all easy to use. Visit http://www.dovepress.com/ testimonials.php to read real quotes from published authors. 